
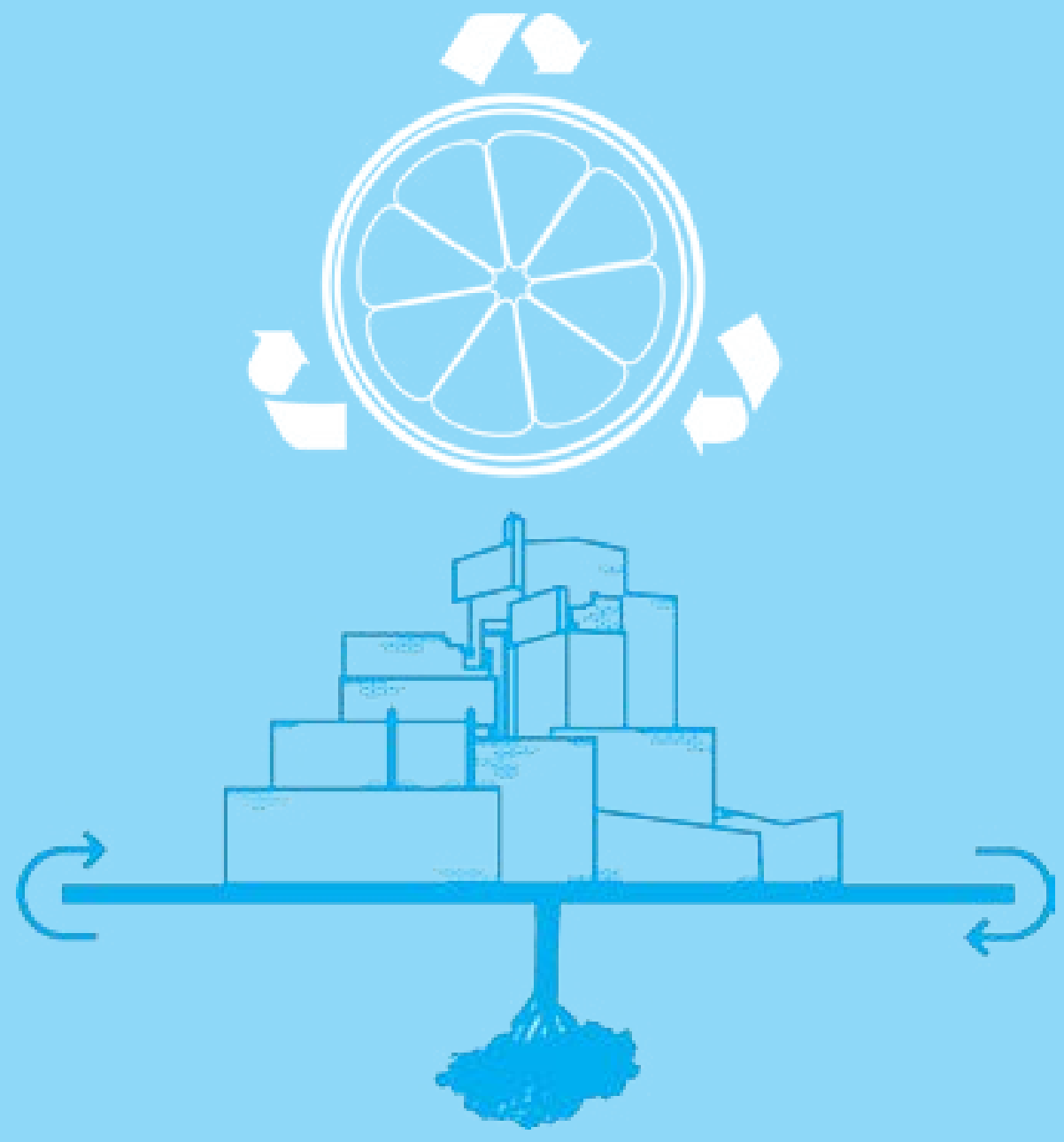

\title{
Oportunidad pedagógica y refrigerio escolar. Una construcción colectiva desde la sistematización de experiencias
}

Educational opportunity and school snack. A collective construction from thesystematization of experiences

Oportunidades educacionais e merenda escolar. A construção coletiva a partir dasistematização de experiências

Francy Angélica Riveros Santa / Rosana Gómez Castro / Pedro Gustavo Sánchez / Camilo Andrés Benavides 


\title{
Oportunidad pedagógica y refrigerio escolar. Una construcción colectiva desde la sistematización de
} experiencias ${ }^{1}$

\author{
Francy Angélica Riveros Santa ${ }^{2}$ \\ Rosana Gómez Castro ${ }^{3}$ \\ Pedro Gustavo Sánchez ${ }^{4}$ \\ Camilo Andrés Benavides Rodríguez ${ }^{5}$
}

1 Este artículo presenta algunas de las comprensiones epistemológicas y avances de la investigación titulada "Oportunidad Pedagógica: refrigerio escolar, convivencia y educación para los Derechos Humanos", realizada por un equipo multidisciplinar de cuatro docentes de la Secretaría de Educación de Bogotá, en el marco de formación docente y la Maestría en Educación de la Universidad Santo Tomás. Bogotá. Colombia. En curso.

2 Docente Secretaría de Educación de Bogotá, Liceo Femenino Mercedes Nariño I. E. D. Licenciada en matemáticas, Universidad Distrital Francisco José de Caldas. Correo electrónico: farsmat@hotmail.com y francyriveros@ustadistancia.edu.co

3 Docente Secretaría de Educación de Bogotá, Colegio Paraíso Mirador I. E. D. Licenciada en Básica con énfasis en matemáticas. Universidad Santo Tomás. Correo electrónico: rouzgo@hotmail.com y rosanagomez@ustadistancia.edu.co

4 Docente Secretaría de Educación de Bogotá, Colegio José Antonio Galán I.E.D. Ingeniero de sistemas, Universidad Católica de Colombia. Correo electrónico: pgs_7@yahoo.com.ary pedrosanchez@ustadistancia.edu.co

5 Docente Secretaría de Educación de Bogotá, Colegio Estrella del Sur I. E. D. Licenciado en Informática, Universidad Minuto de Dios. Correo electrónico: Camilo_benavides@hotmail.com y camilobenavidez@ustadistancia.edu.co

\section{Resumen}

El presente artículo está realizado a partir de una sistematización de experiencias que, desde un enfoque investigativo, permitió que las y los participantes desarrollaran nuevas formas de construir conocimiento. La experiencia buscó comprender: ¿Cómo el refrigerio escolar se convirtió en una oportunidad pedagógica para las y los actores sociales de una comunidad educativa? Las narraciones de dicha comunidad, junto a la nuestra como investigadores, permitieron una reflexión hermenéutica desde una filosofía de la comprensión, desde la cual se planteó una ruta metodológica de tres momentos: reconstrucción histórica, composición del discurso en texto e interpretación.

\section{Palabras clave}

Oportunidad pedagógica, refrigerio escolar, Derechos Humanos.

\section{Summary}

This paper focuses on the systematization of experiences as an investigative approach to generate new ways of building knowledge in the participants. The experience sought to understand how the school meal became a pedagogic opportunity for the staff and students. The narrations from the staff and students as well as from the investigators allowed a hermeneutic reflection based on a philosophy of comprehension, resulting in a methodological route that was developed in three stages: historical reconstruction, speech composition in text form, and interpretation.

\section{Key words:}

Pedagogic opportunity, school meal, Human Rights.

\section{Resumo}

Este trabalho foca na sistematização de experiências com um enfoque investigativo, gerando nos participantes novas formas de construir o conhecimento. A experiência busca compreender como a refeição escolar se transformou em uma oportunidade pedagógica para os profissionais sociais de uma comunidade educativa. As narrativas da comunidade educativa, e nossas como pesquisadores, permitiram uma reflexão hermenêutica desde uma filosofia da compreenção, emergindo uma metodologia que se desenvolveu em três estapas: reconstrução histórica, composição de discurso em texto e interpretação.

\section{Palavras chave: \\ Oportunidade pedagógica, refeição escolar, Direitos Humanos.}




\section{Introducción}

\section{Postura epistemológica}

L presente investigación se estableció desde la sistematización de experiencias, vista como enfoque investigativo de la Educación Popular ${ }^{6}$. El movimiento pedagógico de Educación Popular surge en América Latina como una forma seria, válida y rigurosa de comprender los fenómenos sociales, culturales, políticos y pedagógicos de las prácticas en el aula que, como maestras y maestros, ejercemos cotidianamente. Esto, a partir de dar voz a las y los participantes de los contextos educativos, posicionando la subjetividad como un aspecto relevante en la investigación. Así, abre espacios dialógicos de participación, generando una construcción de saber y conocimiento colectivo que busca interpretar, potenciar y transformar, sin una linealidad preestablecida, los procesos naturales de la vida y de quienes hacemos parte de ella. Por ello Ghiso (2004a), afirma:

Necesitamos, entonces, interrogar y transformar los presupuestos epistémicos y las propuestas metodológicas, por lo que requerimos hacer tránsitos y tomar opciones que nos permitan construir, organizar y recrear conocimientos para la vida. Para ello, asumir posturas que cuestionen las bases de cualquier propuesta investigativa (p. 10).

La sistematización de experiencias permite hacer una revisión de nuestras propias posturas epistémicas y metodológicas, cuestionando como vemos y vivimos la vida. Dicho camino, señala

6 La Educación Popular condensa una multiplicidad de sentidos, a veces contradictorios, tanto a nivel de los discursos, como de las prácticas y de las articulaciones entre ambos. No podría ser de otro modo, pues, como señala Torres (2000a), en sí misma: "la EP no constituye una teoría o cuerpo doctrinal homogéneo. Es una corriente sociopedagógica construida histórica y contextualmente en la que confluye una diversidad de prácticas y discursos diferentes entre sí [...] en cada una de las regiones y países del continente la Educación Popular ha asumido matices particulares: mientras en el Cono Sur estuvo muy ligada a los procesos de lucha contra dictaduras y a los procesos de democratización, en los países andinos como Bolivia y Perú es notorio el acento en lo étnico y lo indígena, en América Central estuvo asociada con los procesos insurrecciónales, en México tuvo su nicho en las iniciativas organizativas independientes que buscaban opciones frente al partido de gobierno". varios aportes de la comunidad de docentes investigadores, posicionando principalmente tres corrientes: "[...] enfoques críticos, empírico-analíticos e histórico- hermenéuticos" (Cifuentes, 1997 a, p. 6). Este último, traza la reflexión del presente trabajo, en el que cobra relevancia la participación y la comunicación de los sujetos, pues parte de una construcción colectiva de conocimiento desde quienes hacen parte de la experiencia.

Según Cifuentes (1997b), investigadores como Torres (2006b), Acevedo (2008) o Londoño (2006), reflexionan sobre tres procesos que deben tenerse en cuenta cuando se realiza este tipo de trabajo: la reconstrucción, la interpretación, y la potenciación. Por su parte, Ghiso (2004b) busca comprender las prácticas sociales desde una mirada humana: en el encuentro con otros es donde realmente se construye conocimiento, pues permite relatar, narrar, la experiencia, reconstruir la historia reflexionando y comprendiendo ¿por qué pasó lo que pasó?; el sentido de la sistematización de experiencias, en las ciencias sociales y en la educación, se da desde la interpretación de las acciones vividas, el lenguaje, y de la puesta en común de actitudes humanas como la indignación, la apropiación del conocimiento, la autonomía y la esperanza.

Por ello encontramos y asumimos la filosofía hermenéutica propuesta en la obra de Ricoeur (2001, 2002, 2004, 2008), quien expone, como supuesto epistemológico, que el ser humano debe reflexionar sobre su propia existencia configurando una identidad de sí mismo que le permita autointrepretarse desde una fenomenología lingüística de las experiencias vividas, así emergerá la temporalidad e historicidad como aspectos centrales de una identidad narrativa : "[...] la hermenéutica aparece en la obra de Ricoeur como un método filosófico a ciertos objetos cuya naturaleza es el lenguaje" (Frey, 2012, p.8).

Narrar la experiencia reconstruyendo la historia y reconstruir la historia narrando la experiencia, en un ciclo hermenéutico, sin una linealidad de hechos y momentos, permite reflexionar

7 El concepto de identidad narrativa en la obra de Ricoeur, es entendido como aprehensión de la vida en forma de relato y determina la comprensión de la vida buena, derivando en una ética. Ricoeur, fundamenta la vida buena en la acción humana y su teleología interna, apoyándose inicialmente en Aristóteles, pero a la vez reconceptualizando la praxis del actuar humano. La acción humana se hace en el tiempo e inscribe la temporalidad en la red del lenguaje y de un contexto institucional. El concepto de acción descansa en las categorías de prácticas, planes de vida y unidad narrativa de vida. La identidad narrativa, desencadena en la medida en que el sujeto de la acción es lector y escritor de su propia vida. (Riveros, 2014a, p.3). 
sobre situaciones naturales de la vida humana desentrañando, desde la identidad de sí mismo, como sujeto, una comprensión que dirige a una filosofía crítica del ser; ésta se comprende y de alguna manera posibilita comprender a las y los otros participantes de la vida y de las experiencias, desplegándose un marco desde la educación en Derechos Humanos que dignifica al ser humano en sí mismo, para reconocer en los otros su humanidad, estableciendo acuerdos y caminos dialógicos de comunicación, educación, y paz.

Sin duda la educación en Derechos Humanos es la vía más racional y prometedora para aprender y tomar conciencia acerca de los valores y principios que enaltecen la dignidad del ser humano, destacar la importancia que han adquirido para regular de mejor forma las relaciones del Estado y la sociedad, comprender de qué manera los Derechos Humanos se han transformado en una fuente de inspiración para construir respuestas adecuadas a la creciente complejidad sociopolítica, a los continuos cambios sociales, a las nuevas demandas que se exigen al sistema educativo y determinar las responsabilidades que corresponden al Estado en este escenario (PLANEDH, 2006, p. 8).

Esta investigación asume una postura epistemológica y metodológica que armoniza dialógicamente la sistematización de experiencias, la hermenéutica riccoeriana y la educación en Derechos Humanos como una reflexión que permita la construcción transformadora de los currículos de las instituciones, brindando la posibilidad de pensar y repensar una educación que forje elementos y dignifique a los niños, niñas y jóvenes del país.

\section{La experiencia}

Esta investigación sistematiza la experiencia de "Doña naranja y sus amigas las rosáceas"» (2010-2015), liderada por dos docentes de una institución educativa distrital ubicada en la parte alta y periférica del barrio Paraíso, Ciudad bolívar, Bogotá. El acceso al sector se da mediante una vía principal en mal estado; también existen otros problemas, consecuencia de la invasión de

\footnotetext{
8 "Doña naranja y sus amigas las rosáceas" es un proyecto liderado por las docentes Lisbeth Lombo y Rosana Gómez del Colegio Paraíso Mirador I. E. D., es reconocido en los Foros Feria local y Distrital (2013) y por la Fundación Terpell (2013).
}

zonas verdes y la contaminación de fuentes hídricas. El barrio se constituyó a partir de un proceso de deforestación y del relleno de las fuentes hídricas, que dio paso a la construcción de edificaciones realizadas por los habitantes, quienes en su gran mayoría son víctimas del desplazamiento forzado.

Además, se presentan problemas ambientales debido al manejo inadecuado de las basuras y el abandono de mascotas en las calles; según el diagnóstico realizado por Sector Hábitat (2011), que considera indicadores como aspectos demográficos, condiciones socio-económicas, vivienda, suelo, servicios públicos, entorno y prevención y control, la localidad ocupa el segundo lugar en la ciudad en cuanto a menor calidad de vida. Algunos niños han sido desplazados de sus sitios de origen y no pueden culminar sus estudios, pues se encuentren fuera del rango de edad establecido; en su mayoría las familias están constituidas por un jefe de hogar, especialmente mujeres, y aquellas que cuentan con padre y madre enfrentan un panorama laboral que evidencia falta de oportunidades?.

Esta realidad no es ajena al Colegio, ya que las y los estudiantes replican los problemas en la institución, principalmente en áreas como convivencia y en la inclusión de los niños en extra edad a las aulas regulares; también se manifiesta la falta de cuidado en el manejo de las basuras producidas durante la hora del refrigerio escolar $\left(\mathrm{PAE}^{10}\right)$. Estas situaciones hacen que las docentes líderes decidan trabajar con los niños y niñas en una propuesta que permita cambiar y mejorar la conciencia ambiental. Al tiempo, motivaron a la creación de un movimiento de participación de la comunidad educativa, en especial los niños y niñas del programa Volver a la Escuela.

"Doña naranja y sus amigas las rosáceas" nace de la observación del contexto y articula una propuesta educativa a partir del consumo apropiado del refrigerio escolar (PAE) y del manejo adecuado de los desechos producidos durante su consumo, para enseñar a la comunidad a darles otros usos, como mermeladas, arequipe y artículos decorativos, como muñecas, velas, monederos, entre otros, a través de la implementación de unidades didácticas que trazan un currículo mediante la integración

9 " $[. .$.$] en este sector encontramos una población perteneciente a los$ estratos socio económicos 1 y 2, de acuerdo al Departamento Administrativo Nacional de Estadística (DANE), en la encuesta calidad de vida Bogotá (2007), la percepción del adulto cabeza de hogar o cónyuge determinó, con un 40,9\% de los encuestados, que los ingresos no les alcanzan para cubrir los gastos mínimos, y en un 53, 5\% solo alcanzan a cubrir estos gastos" (Riveros, Gómez, Sánchez y Benavides, 2015, p.7).

10 Programa de Alimentación Escolar. 
de áreas y el desarrollo de aspectos como el auto-cuidado, el mejoramiento ambiental, la convivencia, la inclusión y la participación de la comunidad.

Estas consideraciones nos llevaron, como grupo de investigación, a reflexionar sobre las narrativas de los participantes para ir construyendo, a partir de una investigación inductiva, incluso contraria a la investigación cualitativa tradicional, conocimiento desde una mirada holística que nos dirige a la toma de decisiones y a construir categorías que surgen a partir de la sistematización. Pretendemos comprender, a través de la sistematización de la experiencia y las narraciones de los docentes, directivos docentes, estudiantes y padres de familia, cómo el refrigerio escolar se convirtió en una oportunidad pedagógica para los actores sociales del colegio, todo, enmarcado en un análisis epistemológico y metodológico con un enfoque hermenéutico.

\section{Metodología}

Para comprender nuestro problema fue necesario proponer una ruta metodológica que armonizara las comprensiones epistemológicas desde una fundamentación riccoeriana, potenciando las narrativas de las y los sujetos participantes, pues es en el relato donde emergen reflexiones relevantes que permiten construir conocimiento en un proceso holístico de investigación inductiva. El proceso de sistematización utilizado fue inductivo, cambiando la lógica del trabajo, pues no partimos de una hipótesis, sino que planteamos un objetivo que determinó lo que pretendimos conseguir; de alguna manera, es un proceso emergente, trazando otra dinámica que va desde lo micro para luego construir y exponer los hallazgos macro. Las categorías van emergiendo, ya que se van encontrando y construyendo a medida que avanza la investigación.

El proceso metodológico se ajusta a un análisis del discurso desde una comprensión hermenéutica; la forma de construir el conocimiento y su interpretación recae en el rigor de las y los investigadores, este proceso subjetivo parte del equipo sistematizador, que toma decisiones, recopila, interpreta y juega con los elementos que dan sentido, para construir colectivamente y finalmente comunicar a la comunidad académica los nuevos conocimientos. El análisis del discurso tiene varios enfoques, de los cuales trabajamos:
Este enfoque investigativo nos ubica en el giro hermenéutico dado en los años setenta, ya que a partir de allí el significado de los actores pasa a ser cardinal. Es gracias a las reflexiones emanadas de allí que los fenómenos sociales pasan a ser vistos como textos que deben ser interpretados, comprendidos. Como lo señala Bruner (1988), la narrativa pasa a ser concebida como una forma de construir realidad. La narrativa se convierte en una cualidad estructurada de la experiencia que se vuelve relato de un yo significante de sus experiencias, un yo que busca permitirse comprender la experiencia vivida, un yo que busca sentidos a cada experiencia dada (Jiménez, 2013, p. 5).

Las narrativas permiten escuchar, relatar, explicar, desde la comprensión, la reconstrucción de los momentos vividos en el transcurso de la vida misma, de las aulas, de las prácticas pedagógicas de los docentes, desde la subjetividad, rompiendo con un paradigma científico sobre la forma de investigar y comprender la realidad en el campo de las ciencias sociales y las Ciencias Humanas. Esta ontología hace que el proceso no parta de una metodología de pasos, sino que se reflexione sobre la epistemología de los mismos, para esto, la ruta desarrolló tres momentos: reconstrucción histórica de la experiencia, composición del discurso del texto "De descripción densa a trama" e interpretación de la experiencia.

\section{Resultados}

La experiencia centrada en las narrativas posiciona a esta investigación desde un giro hermenéutico que dista de la investigación científica "clásica" de las Ciencias Humanas y sociales, permitiéndonos reflexionar sobre nuestro quehacer pedagógico y sobre las intuiciones y decisiones que nos llevan a tomar decisiones en el proceso investigativo; todo, desde un análisis inductivo que pone en tensión nuestras propias posturas epistémicas, las realidades y experiencias que como maestras y maestros vivimos y relatamos, en un diálogo con teorías filosóficas, generando interpretaciones que construyen conocimiento: "De la instancia positivista se pasa a una perspectiva interpretativa, en la cual el significado de los actores se convierte en el foco central de la investigación" (Bolívar, 2002, p. 42).

De esta manera, la narrativa surge a través de la experiencia, que se convierte en relato y se potencia hacia una fenomenología lingüística, componiendo una trama que deriva en una reflexión 


\section{EL REFRIGERIO ESCOLAR: UNA OPORTUNIDAD PEDAGÓGICA ESTRATEGIA: ENTREVISTA 4 A ESTUDIANTE (PARTE 1)}

\author{
Profesor: Estaba Johan y Michael, sí que querían salir a jugar \\ microfútbol, pero el recreo, el patio o la cancha, estaban llenos de \\ cáscaras de naranja, ellos se fueron a hablarle a la profesora. ¿'Tú qué \\ crees que la profesora les dijo?
}

Alumno: La profesora diría que los niños deberían recoger las naranjas para guardarlas y, al mismo tiempo, diría que las necesitaban para hacer las prácticas que estamos haciendo, como los cofres, los monederos y todo eso.

Profesor: Ajá. Y, cuéntenme, después de que ellos las recogen y las organizan; más bien, después de que las recogen, ¿cómo las organizan?

Alumno: Las organizan, ehhhh... las guardan en una bolsa.

Profesor: En una bolsa.

Alumno: Y las mantienen bien, bien, las mantienen bien; no las botan, no las parten; las mantienen limpias.

Profesor: ¿Pero, eso no se pudre? ¿Cómo son las cáscaras de naranja? ¿Cómo afecta que no se dañen?

Alumno: Hay líquidos para que no se pudran.

Profesor: ¿Y uno, cómo las deja secar? ¿Cómo las dejaría secar?

Alumno: La pone a la ventana; cuando haya sol las pone en la ventana.

Profesor: Hmmm. ¿Pero las deja solo en el colegió? ¿Se las puede llevar para la casa?

\section{Figura 1. Entrevista 4 (a estudiante), parte 1}

sobre la identidad narrativa y ética de los propios sujetos participantes. Estos discernimientos, articulados con una ruta metodológica, sientan los relatos de la sistematización.

Después de obtener las estrategias metodológicas que permitieron una reconstrucción de la experiencia, a través de las narrativas, la investigación dirigió otras estrategias, diseñadas especialmente para permitir la comprensión de lo narrado, como se muestra en el siguiente gráfico:

Luego del diseño e interpretación de los relatos, se desarrollaron matrices de interpretación y de tensión, cruzando relatos por medio de subcategorías que posteriormente, en un

\section{Interpretación del texto}

En estos apartes, el estudiante destaca la importancia del trabajo de la docente para enseñarles a los niños a reutilizar las cascaras de naranjas, por otra parte se evidencia que el estudiante tiene un gran grado de apropiación del proyecto.

\section{Categoría emergente}

Intervención docente

Empoderamiento

\section{Sub categoría}

Trabajo de la profesora

Apropiación del proyecto

Reconocimiento

diálogo pedagógico, epistemológico, metodológico y filosófico, se convierten en categorías. Los diseños y análisis delinean una investigación inductiva que va construyendo desde el relato, para luego componer un discurso colectivo que interprete y comprenda las experiencias de la comunidad educativa, llevando a reflexionar sobre ¿Qué pasó?, ¿qué aprendieron?, ¿qué construyeron?, ¿qué transformaron?

Estas elaboraciones llevaron a diseñar una estrategia que presente, de manera armoniosa, el proceso inductivo y categorial de los relatos, sin una linealidad preestablecida, invitándonos a mostrar la experiencia como la vida misma, pues en sus interconexiones de ida y vuelta nos alejamos de la uniformidad positivista de las ciencias sociales para exponer un sentir educativo del conocimiento que construimos en las aulas; por ello, a partir de estas reflexiones, construimos el siguiente mapa hermenéutico:

Las categorías son producto de la composición del discurso; a través de los relatos, las construcciones llegan a ser intercambios, una discusión colectiva entre pares y con la comunidad educativa, que llevan a reflexiones que no solo abordan un concepto, sino que dirigen hacia comprensiones de sentido pedagógico y dan respuesta al cuestionamiento inicial de esta investigación. Los principales hallazgos se exponen a continuación ${ }^{11}$ :

11 Otras comprensiones de categorías están en proceso de reflexión y 


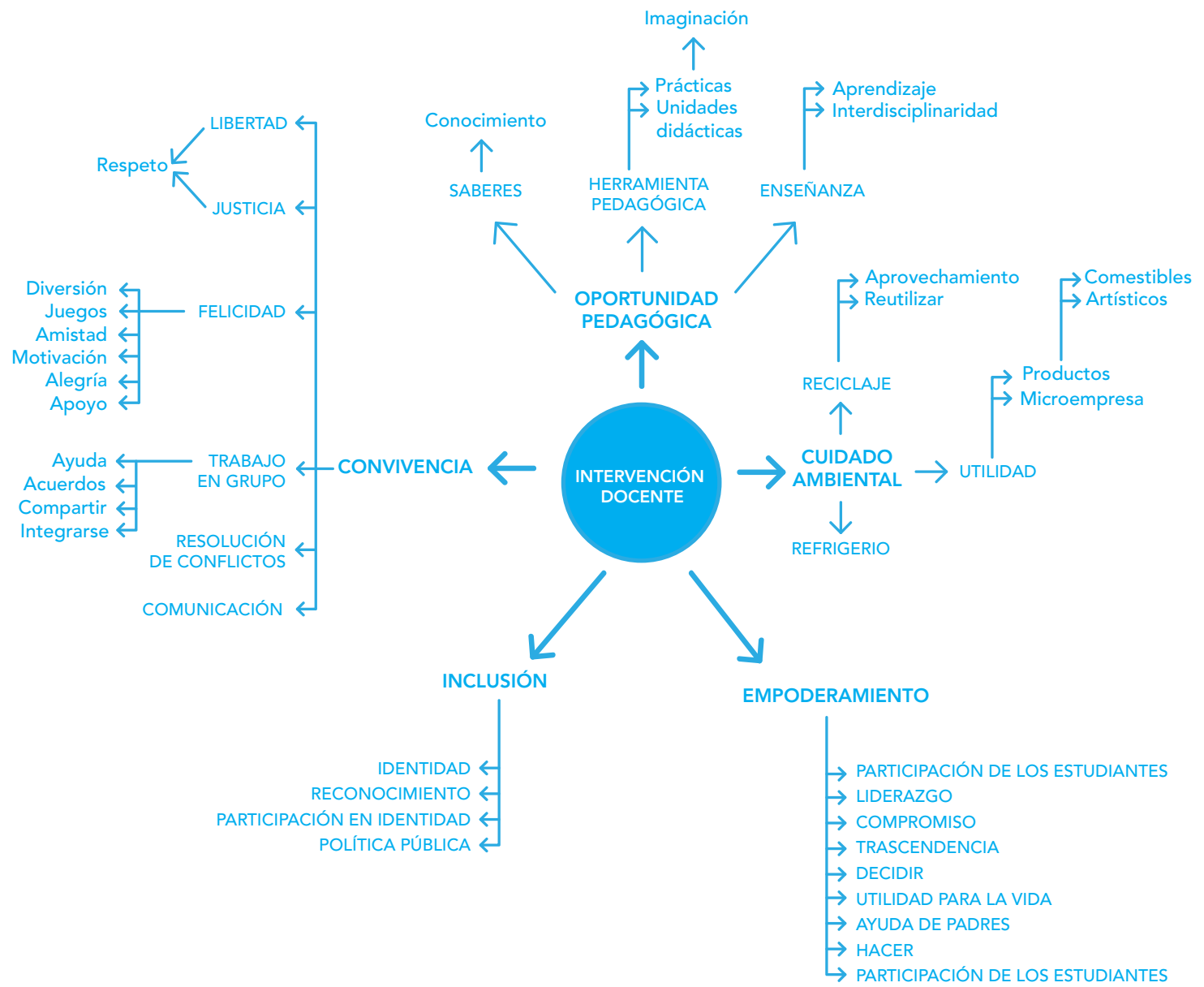

Figura 2. Mapa hermenéutico

\section{Oportunidad pedagógica}

La oportunidad es un momento que me hizo reflexionar acerca de cómo se utilizaba esa palabra, o sea, después de que nosotros en un momento dado establecimos que la razón, el objetivo de nuestra sistematización, iba ser la oportunidad pedagógica; entonces yo me puse a buscar y a observar en dónde era que más se utilizaba la palabra oportunidad. Entonces, en la parte comercial se usa muchísimo, porque todo se piensa que es un negocio en la vida, pero después de reflexionar me di cuenta de

discusión, las presentaremos posteriormente, cuando se termine esta investigación. que oportunidad no es solamente un negocio; no más, yo me levanté por la mañana, entonces tengo una nueva oportunidad de hacer algo nuevo en mi vida, de ofrecer algo nuevo, de conseguir algo nuevo, de vivir un día nuevo, entonces oportunidad no es el simple hecho de buscar un negocio o buscar una rentabilidad, sino el hecho de poder vivir un día más ${ }^{12}$.

La Real academia de la lengua (s. f.) define el término oportunidad (Del latín opportunitas, -ātis), como: 1. f. Sazón, coyuntura, conveniencia de tiempo y de lugar; 2. f. pl. Sección de un comercio en la que se ofrecen artículos a un precio más bajo del 12 Comunicación personal con Sánchez, 10 de abril de 2014. 
que normalmente tienen. Por su parte, Hugo Ernesto V., teólogo, manifestó que: "Considerar que las oportunidades son eso oportunidades (Del latín opportunitas, -ätis). Que no es más que una conveniencia de tiempo y de lugar. No siempre convienen y no siempre corresponden".

El término también acepta expresiones como las de Churchill (s. f): "Un optimista ve una oportunidad en toda calamidad, un pesimista ve una calamidad en toda oportunidad". Popularmente es conocida la expresión: "la ocasión la pintan calva", que proviene de la mitología romana y se refiere a la diosa Ocasión. Luego de ver las descripciones del término, podemos entender que en el proyecto se entendió como algo temporal, ocasional y coyuntural.

¿Qué entendemos por oportunidad pedagógica? En el contexto educativo e investigativo encontramos otras definiciones que nos acercan al concepto; ideas como innovación pedagógica e iniciativa pedagógica, entendidas como constructos de teorías trabajadas en las aulas de clase y en los programas educativos, permiten una reflexión enmarcada en nuestras propias vivencias pedagógicas, como una directriz, una curiosidad, un tema a tratar, una teoría a construir que casi siempre mantiene una distancia del maestro como sujeto investigador de su propio saber, exigiendo estructuras uniformes de linealidad de procesos.

Así, encontramos que la Secretaría de Educación del Distrito (2015), define la Iniciativa Pedagógica como todas aquellas prácticas, acciones, propuestas, proyectos que las escuelas definen y desarrollan colectivamente a partir de una problemática, un interés o una necesidad, vinculadas directamente con procesos de enseñanza y aprendizaje. Para llevarla a cabo es necesario el acompañamiento pedagógico, la financiación, la experiencia en formulación de proyectos, la vinculación a programas de política pública, un plan de trabajo y recursos y espacios de reflexión que permitan mejorar procesos de enseñanza-aprendizaje.

En este contexto, las Iniciativas Ciudadanas de Transformación de Realidades (INCITAR, 2014), se constituyen como un programa para la creación de oportunidades de aprendizaje que lleven a los miembros de una comunidad a generar acciones pedagógicas que construyan ciudadanía. A ellas se suman otros casos que siguen esta línea en Latinoamérica, como el Programa Integral para la Igualdad Educativa (2010), en Argentina, que describe las iniciativas pedagógicas como un conjunto de decisiones relacionadas con los procesos de enseñanza, que cobran en la actualidad una relevancia sustantiva.
Desde esa perspectiva definimos la Iniciativa Pedagógica (IP) como parte del Proyecto Educativo (PE), que se constituye a partir de un conjunto de acciones que los adultos acuerdan realizar en las propuestas de enseñanza, con el propósito de mejorar los aprendizajes de los alumnos en el ámbito escolar (p. 8).

En el caso de innovación pedagógica, recopilamos varias interpretaciones que nos acercaron a la noción de producción sistémica, la cual guarda relación con conceptos de productividad y eficacia, indicadores de gestión propios de los tiempos modernos. Según Fernández:

[...] una innovación, para ser considerada como tal, necesita ser duradera, tener un alto índice de utilización y estar relacionada con mejoras sustanciales de la práctica profesional, esto establecerá la diferencia entre simples novedades (cambios superficiales) y la auténtica innovación (2010, p. 31).

Por otra parte, Blanco y Messina, en el informe de la Unesco para América Latina, concluyen que:

Innovación supone transformación y cambio cualitativo significativo, no simplemente mejora o ajuste del sistema vigente. Si bien la innovación implica cambio, existe un consenso entre los diferentes autores respecto a que no todo cambio es una innovación. La innovación supone una transformación, un cambio cualitativo significativo respecto a la situación inicial en los componentes o estructuras esenciales del sistema o proceso educativo. La innovación supone partir de lo vigente para transformarlo, por lo tanto, parte de un cambio en las estructuras y concepciones dadas (2000, p. 63).

Este panorama nos permite ver la Oportunidad Pedagógica como un concepto cercano y distante de las iniciativas e innovaciones pedagógicas definidas anteriormente. Busca comprender una elaboración de acuerdo con el trabajo de sistematización; en ese trabajo resulta relevante un proceso de descolonización de los maestros investigadores y participantes del mismo, discerniendo, debatiendo, reflexionando y construyendo comprensiones a través de nuestras propias posturas epistémicas: 
[...] entonces, construir en este concepto de oportunidad pedagógica es nuevo para nosotros, porque nos invita a pensar; no es una formulación ya programada, porque fácilmente podríamos decir que es una innovación o una iniciativa, pero se diferencia porque la oportunidad parte de una circunstancia [...] las dos profesoras no la evidenciaron en su momento. Se dieron cuenta de que por algún lado podían trabajar, pero que, como dice Camilo, se dio en cierto colegio, de pronto en el colegio donde trabajamos o donde trabaja Pedro se dan otras oportunidades, pero es el docente o la docente quien debe encaminarla para que esa oportunidad se convierta en oportunidad pedagógica; ahí me parece valiosa esa armonía, encontrar equilibrio entre las dos posturas ${ }^{13}$.

Es así como en este recorrido de construcción nace la Oportunidad. Pedagógica, la cual interiorizamos como un acontecimiento, suceso, práctica cotidiana, naturalizada en un tiempo y un lugar determinado por las y los integrantes de un contexto educativo, que luego de ser reflexionada por un colectivo de docentes y/o directivos docentes, incide en acciones, no necesariamente sistemáticas, pero sí rigurosas, llevando a la comunidad educativa a vivir una experiencia que le permita reconocerse a sí misma desde la individualidad, para luego reconocer a las y los otros como sujetos de un grupo social que se construye a través de procesos de enseñanza y aprendizaje.

\section{Conclusiones}

La oportunidad pedagógica mencionada, es una construcción colectiva de conocimiento que surge desde la sistematización, desarrollando un diálogo entre y con la comunidad educativa, que a través de sus relatos permitió la reconstrucción de la experiencia, para posteriormente componer un discurso que al ser interpretado por las y los investigadores, en un diálogo hermenéutico, potenció reflexiones sobre las prácticas que ejercemos y las posturas epistemológicas que concebimos, discerniendo en la categoría descrita.

Es necesario reconocer que aunque conocemos otros conceptos, elaborados desde otras teorías, como la innovación pedagógica, la iniciativa educativa o la iniciativa pedagógica, éstos se alejan de nuestras opiniones, pues no desarrollan un sistema riguroso que enmarque la vida de las aulas en procesos eficaces de control y de uniformidad, cíclicos, que respondan a evaluaciones planeadas supervisadas, así mismo, no consideramos que esto suceda en una planeación del paso a paso.

Las oportunidades se dan en un marco espontáneo de la vida misma en las aulas de clase; sin embargo, cada docente toma la decisión de llevar la simple oportunidad hacia una oportunidad pedagógica; entonces, sin lugar a dudas, el o la docente son uno de los protagonistas de la acción, ya que, de alguna manera, son quienes interiorizan naturalmente sus prácticas sociales, culturales, políticas, educativas, epistemológicas, didácticas, entre otras, en la oportunidad pedagógica; lo cual los lleva a determinar los procesos de enseñanza y aprendizaje que incidan en los currículos de los establecimientos educativos y, en consecuencia, en la transformación de la vida de los y las estudiantes.

En este sentido es que la historia de la educación destaca la importancia del docente, a través de los marcos normativos nacionales e internacionales; los lineamientos que surgen de la academia en programas de pregrado de las licenciaturas; el análisis de investigadores educativos; la reflexión de los sindicatos; y, en general, la mirada de la sociedad. Todo ello da como resultado investigaciones y propuestas que son objeto de discusión y reflexión sobre la tarea de los y las docentes. En Colombia se reconoce al docente como uno de los ejes centrales de la educación, responsable de varias funciones, así, el Decreto 1278 de Junio 19 de 2002, cita en su Artículo 5:

Las personas que desarrollan labores académicas directa y personalmente con los alumnos de los establecimientos educativos, en su proceso enseñanza-aprendizaje, se denominan docentes. Estos también son responsables de las actividades curriculares no lectivas complementarias de la función docente de aula, entendidas como administración del proceso educativo, preparación de su tarea académica, investigación de asuntos pedagógicos, evaluación, calificación, planeación, disciplina y formación de los alumnos, reuniones de profesores, dirección de grupo, actividades formativas, culturales y deportivas, atención a los padres de familia y acudientes, servicio de orientación estudiantil y actividades vinculadas con organismos $\mathrm{o}$ instituciones del sector que incidan directa o indirectamente en la educación.

13 (Riveros, comunicación personal, 10 de abril de 2014b). 
Desde el marco internacional, el Informe de Seguimiento de Educación para Todos (EPT, 2013), establece que la tarea docente debe ser atendida para lograr una educación de calidad. Entonces, podemos inferir que la intervención del docente es necesaria para que se dé la oportunidad pedagógica, pues desde la observación, la intuición, su saber practico, el diálogo con pares, con sus estudiantes y con la comunidad, se le permite sentir y otorgar su sello pedagógico a las oportunidades que encuentra en la vida misma de las aulas, determinando la relevancia de guiar un camino que lo lleve a desarrollar propuestas pedagógicas, proyectos, reflexiones, debates y transformaciones en currículos generadores de cambio.

La intervención docente, como uno de los ejes centrales de la oportunidad pedagógica, requiere de profesores empoderados de su práctica pedagógica, naturalizando su saber y comprometiéndose con la investigación educativa desde las mismas aulas:

\footnotetext{
Ser un maestro-investigador o una maestra-investigadora es en sí mismo un acto rebelde contra los rumbos tradicionales de investigación. Él o ella dejan de ser un mero lector de estudios educativos que le dicen qué hacer en su aula. [...] Tanto docentes como investigadores tienen el poder y el potencial de promover cambios en distintos niveles: personalmente, en sus aulas, en la escuela, en la comunidad o en el campo educativo en general (Uttech, 2005, p. 148).
}

El empoderamiento de las practicas pedagógicas, del saber colectivo generado en las aulas por las y los docentes, potencia una intervención desde la libertad, la crítica, la confianza, la autonomía, que entiende todos estos aspectos como algo esencial que lleva a encontrar oportunidades pedagógicas: "[...] la expansión de la libertad, la autoridad y el poder de la persona sobre los recursos y las decisiones que afectan su vida (Chaná, 2012, p. 1).

Entonces, la Oportunidad Pedagógica se articula armoniosamente con la intervención docente desde un empoderamiento de su saber como maestro, maestra, investigador, investigadora, en una reflexión crítica de la vida, de las tensiones que encontramos y que permiten potenciar construcciones colectivas de cambio y transformación en las aulas y, en consecuencia, en la sociedad.

Sobre la experiencia se puede afirmar que desde algo tan natural en las instituciones educativas distritales, como el con- sumo del refrigerio escolar, se construyó toda una propuesta que permitió desarrollar enseñanzas y aprendizajes de diversos órdenes, posibilitando una educación en Derechos Humanos. El refrigerio escolar no es solo comida, es un derecho de los niños, niñas y jóvenes; esto implica enseñar su consumo responsable y adecuado, analizando el derecho a la educación de calidad. También supone una formación que contemple el bienestar de los seres humanos desde la convivencia y el medio ambiente, reflexionando sobre problemas como el hambre de las poblaciones vulnerables; esto nos posiciona como docentes corresponsables de una formación en el derecho mismo.

La articulación de políticas públicas que garanticen una educación de calidad es una obligación de todas y todos. Docentes empoderados, veedores de la educación, críticos, propositivos, autónomos, hacen que desde las aulas sea posible desarrollar investigaciones y aportes relevantes a la educación, y que éstas deriven en un modelo social y humano de la vida misma. El refrigerio escolar se convirtió en una oportunidad pedagógica para esta comunidad educativa, la invitación es a pensar: ¿cuál es su oportunidad pedagógica? 


\section{Referencias}

Barnechea, M., y Morgan, M. (2010). La sistematización de experiencias: producción de conocimientos desde y para la práctica. Retos, 15, pp. 97-107. Obtenido desde http://www. cepalforja.org/ sistematizacion/cgi-bin/blosxom.cgi/reflexion_teorica

Benavides, C., Gómez., R., Riveros., F., y Sánchez., P. (2014). El refrigerio escolar: una oportunidad pedagógica. Trabajo de Grado. (No publicado). Universidad Santo Tomás. Bogotá.

Blanco, R., y Messina, G. (2000). Estado del arte sobre las innovaciones en América Latina. Bogotá: Convenio Andrés Bello.

Bolívar, A. (2003). ¿De nobisipsissilemus? Epistemóloga de la investigación biográfico-narrativa en educación. Revista electrónica de Investigación Educativa, 4/19. Obtenido desde http://redie.ens. uabc.mx/vol4nol/contenido-bolivar.htm

Borjas, B. (2003). Metodología para sistematizar prácticas educativas: por las ciudades de Ítalo Calvino. Caracas: Federación Internacional de Fe y Alegría.

Chaná P. (s.f.). El "empoderamiento": una nueva dimensión en la relación médico-paciente. Santiago: Universidad de Santiago de Chile, Clínica Alemana de Santiago. Obtenido desde http://www. scielo.cl/pdf/rmc/v140n3/art20.pdf

Cifuentes, R. (1997). Sistematización de la práctica en trabajo social. Bogotá: Lumen.

Conde, F. (1994). Las perspectivas metodológicas cualitativa y cuantitativa en el contexto de la historia de las ciencias. En Delgado, J., y Gutiérrez, J. Métodos y técnicas cualitativas de investigación en Ciencias Sociales. Madrid: Proyecto Editorial Síntesis, Psicología.
De Sousa Santos, B. (2011). Introducción: Las epistemologías del Sur. Obtenido desde http://www.boaventuradesousasantos.pt/media/INTRODUCCION_BSS.pdf

Díaz, V. (2006). Formación docente, práctica pedagógica y saber pedagógico. Laurus. Revista de Educación, 12, pp. 88-103. Obtenido desde http://www.redalyc.org/pdf/761/761 09906.pdf

Departamento Administrativo Nacional de Estadística (DANE). (2007). Encuesta calidad de vida Bogotá. Obtenido desde http://www.dane.gov.co/index.php/es/estadisticas-sociales/calidad-de-vida-ecv/87-sociales/calidad-de-vida/3894-encuesta-calidad-de-vida-bogota-2007

Espada, C. (2010). La innovación educativa. Revista Digital Eduinnova, 23, pp. 30-33. Obtenido desde http://www.eduinnova.es/ junio2010/innovacion.pdf

Frey, D., y Stricker, N. (2013). Escritos y Conferencias 2: Hermenéutica. México: Siglo XXI.

Guiso, A. (2004). Entre el hacer lo que se sabe y el saber lo que se hace. Dimensión Educativa. Sistematización de Experiencias -Propuestas y debates-. Bogotá: D.E.

Jara, O. (2012). Sistematización de experiencias, investigación y evaluación: aproximaciones desde tres ángulos. Revista internacional sobre investigación en educación global y para el desarrollo, 1, pp. 56-70. Obtenido desde http://educacionglobalresearch. net/wp-content/uploads/02A-Jara-Castellano.pdf

Jiménez, D. (2013-Septiembre). Problemáticas que las demandas de participación de grupos sociales realizan utilizando el discurso 
de la diferencia y exigen la transformación de educación formal de Colombia. Ponencia presentada en II Congreso Internacional en Temas y Problemas en Investigación en Educación, Sociedad, Ciencia y Tecnología. Bogotá. Obtenido desde http://soda.ustadistancia.edu.co/enlinea/paginaimagenes/PRESEN TACIONESyPONENCIAS/Memorias\%20Ponencias/Bogota/Educacion\%20 y\%20derechos\%20humanos/Mesa\%202\%20Septiembre\%2020/ David\%20Andr\%E9s\%20Jim\%E9nez.pdf

Ministerio de Educación. (2002). Decreto 1278. Obtenido desde http://simac.org.co/admin/ files/5ebb7f79.pdf

Ministerio de Educación, Presidencia de la Nación. (2010). Programa integral para la igualdad educativa. Iniciativas Pedagógicas. Obtenido desde http://www.entrerios.gov.ar/ CGE/2010/primaria/files/2011/02/Iniciativas-Pedagogicas.pdf

Plan nacional de educación. (2006). Un pacto social por la educación. Obtenido desde http: //www.sedbogota.edu.co/archivos/ Nuestra_Entidad/VERSION_FINAL_PNDE_INTERACTIVA. pdf

Ricoeur, P. (2001). Del texto a la acción. Ensayos de hermenéutica II. Buenos Aires: FCE.

Ricoeur, P. (2002). Del texto a la acción. Ensayos de Hermenéutica II. México: F.C.E.

Ricoeur, P. (2004). Tiempo y narración I. México: Siglo XXI.

Ricoeur, P. (2008). La memoria, la historia y el olvido. México: Siglo XXI.

Riveros, F. (2014). Sí mismo como otro. Sexto estudio: el sí y la identidad narrativa II. Entre describir y prescribir. Narrar. (No publicado). Bogotá: Universidad Santo Tomas.

Secretaría de Educación del Distrito. (2014-Junio). Incitar: iniciativas ciudadanas de transformación de realidades. Obtenido desde http://www.educacionbogota.edu.co/index.php?option=com_content $\&$ view $=$ article \&id=2434:incitar\&catid=16:temas-estrategicos

Torres, A. (1998-Octubre). La sistematización de experiencias educativas: reflexiones sobre una práctica reciente. Ponencia presentada en el tercer Congreso Iberoamericano y Caribeño de agentes de desarrollo sociocultural y comunitario. La habana, Cuba.
Torres, A. (2000). Ires y venires de la Educación Popular en América Latina. La Piragua: Revista latinoamericana de educación y política. 18, pp. 19-29. Obtenido desde http://www .oei.es/n5069. htm

Unesco. (2014). Informe de seguimiento de la Educación para todos en el mundo. Obtenido desde http://unesdoc.unesco.org/ images/0022/002261/226159s.pdf

Uttech, M. (2005). ¿Qué es la investigación-acción y qué es un maestro investigador? XXI Revista de educación, 8, pp. 139-150. 